\title{
Intrinsic bent DNA sites in the chromosomal replication origin of Xylella fastidiosa 9a5c
}

\author{
F. Gimenes ${ }^{1}$, F. de S. Gouveia ${ }^{1}$, A. Fiorini² and M.A. Fernandez ${ }^{1}$ \\ ${ }^{1}$ Departamento de Biologia Celular e Genética, Universidade Estadual de Maringá, Maringá, PR, Brasil \\ ${ }^{2}$ Centro Universitário de Maringá, CESUMAR, Maringá, PR, Brasil
}

Correspondence to: M.A. Fernandez, Departamento de Biologia Celular e Genética, Universidade

Estadual de Maringá, Av. Colombo, 5790, 87020-900 Maringá, PR, Brasil

Fax:+55-44-3261-4893. E-mail: aparecidafernandez@gmail.com

\begin{abstract}
The features of the nucleotide sequences in both replication and promoter regions have been investigated in many organisms. Intrinsically bent DNA sites associated with transcription have been described in several prokaryotic organisms. The aim of the present study was to investigate intrinsic bent DNA sites in the segment that holds the chromosomal replication origin, oriC, of Xylella fastidiosa 9a5c. Electrophoretic behavior analyses, as well as in silico analyses of both the 2-D projection and helical parameters, were performed. The chromosomal segment analyzed contains the initial sequence of the rpmHgene, an intergenic region, the $d n a A$ gene, the oriC sequence, and the 5' partial sequence of the $d n a N$ gene. The analysis revealed fragments with reduced electrophoretic mobility, which indicates the presence of curved DNA segments. The analysis of the helical parameter ENDS ratio revealed three bent DNA sites $(b 1, b 2$, and $b 3)$ located in the $r p m H$-dnaA intergenic region, the $d n a A$ gene, and the oriC 5' end, respectively. The chromosomal segment of $X$. fastidiosa analyzed here is rich in phased AT tracts and in CAnT motifs. The 2-D projection indicated a segment whose structure was determined by the cumulative effect of all bent DNA sites. Further, the in silico analysis of the three different bacterial oriC sequences indicated similar negative roll and twist $>34.00^{\circ}$ values. The DnaA box sequences, and other motifs in them, may be associated with the intrinsic DNA curvature.
\end{abstract}

Key words: Intrinsic bent DNA; Prokaryotic replication origin; oriC; Xylella fastidiosa

Research supported by CNPq (No. 473966/2003-4/501381/2003-1) and the Academy of Science for the Developing World, TWAS (RGA 00-265 RG/BIO/LA). F.S. Gouveia was the recipient of a fellowship from CAPES.

Received September 25, 2007. Accepted March 5, 2008

\section{Introduction}

After the structure of DNA was solved (1), it was proposed that DNA molecules could exist in several structural conformations (2). The discovery of alternative structures, such as curvature in the promoter and replication origins that were either induced by the intrinsic DNA sequence or produced by DNA flexibility, permitted the establishment of a relationship between structure and function (3).

Intrinsic bent DNA sites are usually caused by adenine/ thymine-rich tracts that are in phase with helical repeat $\sim 10.5$ base pairs (bp) (4). Alternatively, flanking sequences can have a significant influence on DNA conformation; some curvatures have been described in the absence of $A$ / $\mathrm{T}(5)$.
Curved DNA sequences have been found at regulatory sites, including prokaryotic (6) and eukaryotic (7) gene promoters. It is known that the intrinsic curvature of DNA plays an important role in cellular processes such as nucleosome compaction (8), recombination processes (9), fragile sites (10), tandem repeated sequences (11), replication origins (12,13), Scaffold/Matrix Attachment Regions (S/MARs) (14) and MARs associated with replication origins $(13,15)$.

Investigation of the nucleotide sequences of Archaebacteria has revealed that genomic DNA sequences of replication initiation contain similarities, as described for eukaryotes (16). The replication origins of prokaryotes and lower eukaryotes are located in specific DNA sequences that are always found at the same site of the chromosome. 
These sequences are by definition segments of DNA necessary and sufficient for molecular replication (17). Specific protein-binding sequences in prokaryotic replication sites can present conserved structural domains such as curved DNA, which plays an important role in DNA replication (18).

Xylella fastidiosa 9a5c is a Gram-negative microorganism that causes citrus variegated chlorosis disease in citrus trees (19). The complete genome sequence of strain $9 \mathrm{a} 5 \mathrm{c}$ has been reported (20). The replication origin, which controls the duplication of the single chromosome of $X$. fastidiosa, contains AT-rich segments, five putative DnaA box sequences (R1-R5), and a box of 24 nucleotides with eight ACC repetitions (21). The box of 24 nucleotides replaces the traditional box of 13 nucleotides reported for the Escherichia coli chromosome (22). These regions are important for both protein-binding and the opening of DNA in the bacterial replication process. A report, which states that the genetic transformation of $X$. fastidiosa 9 a5c is possible only with plasmids building from the chromosomal $X$. fastidiosa replication origin (21), suggests that this oriC has an essential feature that other oriC lack. Thus, the evidence implies that this chromosomal origin is essential for the $X$. fastidiosa transformation system and is structurally different from the $E$. coli origin consensus. Thus, the aim of the present study was to map intrinsic bent DNA sites in the $X$. fastidiosa replication origin by electrophoretic behavior and by in silico analyses. In order to compare these results with other bacterial replication origins, we performed in silico analyses as 2-D projections and evaluated the roll and twist helical parameters in the oriC sequences of the $X$. fastidiosa strain Temecula1, Bacillus subtilis, and $E$. coli.

\section{Material and Methods}

\section{DNA fragments}

The recombinant plasmids p16KdAori and p16Kori were kindly provided by Patrícia B. Monteiro (Fundação para a Defesa da Citricultura, Fundecitrus, Araraquara, SP, Brasil). These plasmids contain DNA fragments that include the replication chromosomal origin of $X$. fastidiosa (21). The clone $\mathrm{p} 16 \mathrm{KdAori}$ was constructed in a pBluescript (pBS) plasmid, along with an insert of 4139 bp that contains the $X$. fastidiosa ribosomal operon promoter (rop), the gene for resistance to kanamycin $\left(k^{r}{ }^{r}\right)$, and a fragment of $1892 \mathrm{bp}$. This fragment contains the initial sequence of the rpmH gene, a 141-bp intergenic region, the dnaA gene, the oriC region, and 24 initial nucleotides of the dnaN gene. The oriC fragment is restricted to the intergenic region dnaA-dnaN with 296 bp. The p16Kori clone contains the oriC within a 364-bp fragment. The recombinant plasmids
p16KdAori and p16Kori were transformed in $\mathrm{DH} 5 \alpha$ E. coli competent cells (23), and the plasmid DNA was purified by the CTAB method (24) and/or by alkaline lysis (23). After purification, the plasmids were cleaved with different restriction enzymes according to manufacturer instructions.

\section{Electrophoretic analysis of fragments}

The restriction subfragments were electrophoresed on $1 \%$ agarose gels (AGA) at room temperature and on $6 \%$ polyacrylamide (PA) gels at $4^{\circ} \mathrm{C}$ without ethidium bromide $(\mathrm{EtBr})$. To confirm the presence of bent DNA, the samples were incubated overnight with $1 \mu \mathrm{g} / \mathrm{mL} \mathrm{EtBr}$ and electrophoresed on previously run 6\% PA gels with $1 \mu \mathrm{g} / \mathrm{mL} \mathrm{EtBr}$ in the running buffer $(\mathrm{PA}+\mathrm{EtBr})$. EtBr acts locally between base pairs, abolishing the intrinsic DNA curvature and straightening the fragments (25). All gels were run in $1 \mathrm{X}$ TBE buffer (45 mM Tris-borate, $1 \mathrm{mM}$ EDTA, pH 8.0), molecular weight marker 1-kb Ladder Invitrogen, stained after running with $0.1 \mu \mathrm{g} / \mathrm{mL} \mathrm{EtBr}$, and documented with a UVP Biolmaging System, USA, under UV light. The gel concentration, running time, and running voltage were adjusted relative to the size of the fragments analyzed. The mobility pattern of each fragment was obtained by the calculation of the $R$-value (ratio of apparent/real fragment size observed in each gel system). $R$-values between 0.90 and 1.09 indicate no alterations in mobility, those higher than or equal to 1.10 indicate reduced mobility, and those lower than 0.90 indicate fast mobility $(14,26)$.

\section{In silico analysis of the curvature}

In silico analysis of the DNA sequence curvature provided results that are compatible with electrophoretic data (18). In the present study, we used the dinucleotide wedge model of Bolshoy et al. (27) to predict the 3-D path of the 1892-bp DNA segment. The 2-D projection and structural parameters were calculated by Map15a and 3-D15m1 softwares (28), using the algorithm of Eckdahl and Anderson (18) and the helical parameters of Bolshoy et al. (27), as described by Pasero et al. (11) and Marilley and Pasero (29). Each plotted value takes into account the contribution of the surrounding nucleotides. Putative bent segments were identified using the ENDS ratio, which describes the ratio of the contour length of a fragment helical axis to the shortest distance between the fragment ends. The twist angle (horizontal rotation between 2 consecutive bp), variations of the roll angle (which estimates the rolling-open of the base pairs along their long axes) and $\Delta G$ values, which predict the stability of the DNA duplex, were calculated with the Map15a software. These calculations used a 120bp window width and a 10-bp step for the 1892-bp segment, as well as a 30-bp window width and a 10-bp step for 
the oriC segments from $X$. fastidiosa 9a5c (NC_002488), $X$. fastidiosa strain Temecula1 (NC_004556), Bacillus subtilis (NC_000964), and E. coli (AC_000091).

By convention, if base pairs open toward the minorgroove side, the roll angle is positive. Conversely, negative roll angles that cause a narrow minor groove suggest a bent region (30). The 3-D15m1 software was used to obtain both the 2-D projection of the 3-D fragment trajectory and the $\Delta \mathrm{G}$ values.

\section{Results}

\section{Electrophoretic mobility}

Restriction fragments of the p16KdAori and p16Kori clones were analyzed by electrophoresis on AGA gels, PA gels, and PA + EtBr gels. On PA gels, the migration of DNA fragments was dependent on both size and structure.
Fragments containing bent DNA segments migrated through the mesh of this gel with lower or higher apparent molecular sizes than expected from the fragment length. In contrast, the migration of fragments through AGA gels, which form an irregular mesh, is dependent only on their size. The PA gels confirm the presence of bent DNA sites in fragments. EtBr on these gels intercalates between the DNA base pairs and the fragments, abolishing DNA curvature, and thus the fragments have a regular mobility in this gel. In the p16KdAori clone, the restriction enzymes EcoRI, $B a m H I$ and $X b a l$ did not cut the rop promoter sequence or the $k n^{r}$ gene, keeping the remaining insert linked to the pBS plasmid (Figure 1, black arrow on gels). Double digestion with the $B a m H I+X b a l$ produced two subfragments: one 997-bp fragment containing the initial sequence of the $r p m H$ gene, the 141-bp intergenic region, and part of the $d n a A$ gene, and a second 895-bp fragment that contains
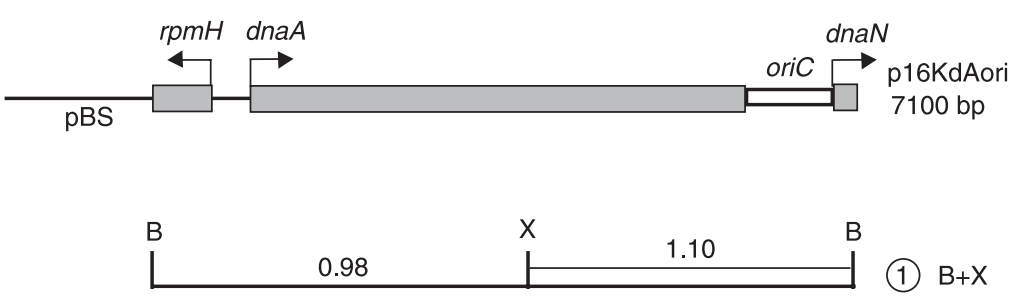

(1) $B+X$
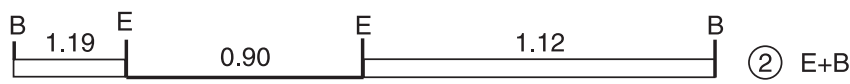

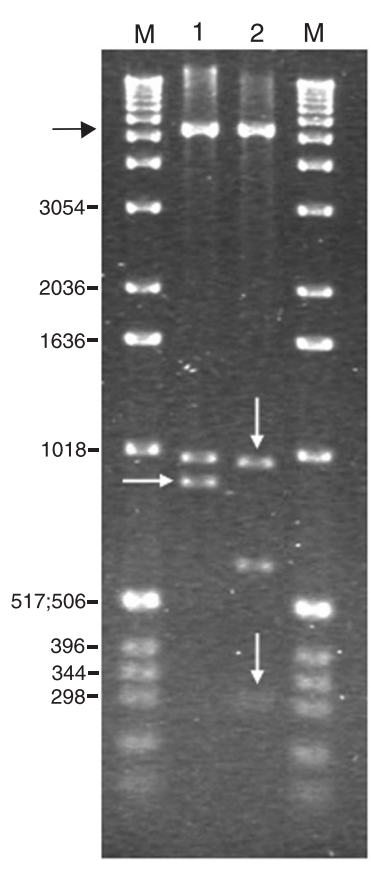

AGA

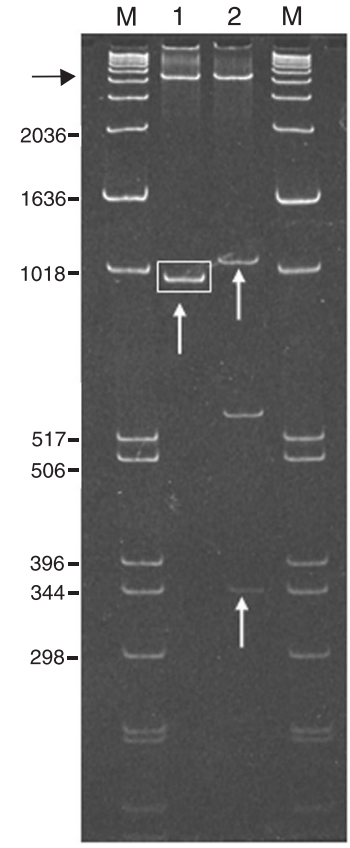

PA

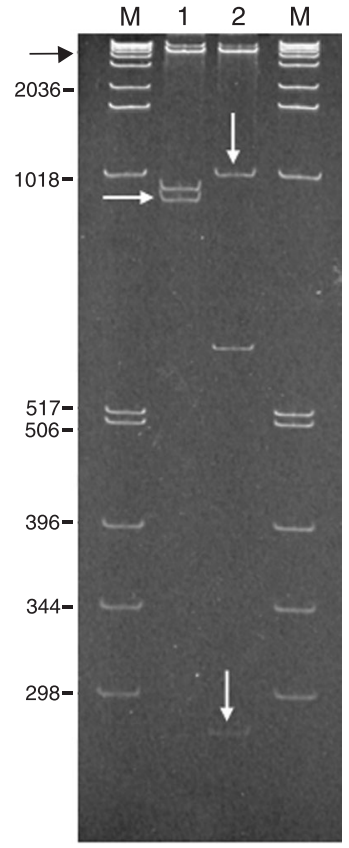

$\mathrm{PA}+\mathrm{EtBr}$
Figure 1. Analysis of the electrophoretic mobility of the oriC chromosomal segment from Xylella fastidiosa 9a5c. The restriction map of the p16KdAori (7100 bp) clone is at the top. The numbers above each fragment are the $R$-values. White vertical and horizontal arrows indicate fragments with reduced mobility, with $R$-values $\geq 1$.10; black horizontal arrows represent a fragment that contains the ribosomal operon promoter (rop) and the gene for resistance to kanamycin $\left(\operatorname{kan}^{r}\right)$, which remain linked to the plasmid pBluescript ( $p B S$ ) in this restriction enzyme cleavage. $M=$ molecular weight marker 1-kb Ladder; AGA $=1.0 \%$ agarose gel; $\mathrm{PA}$ and $\mathrm{PA}+\mathrm{EtBr}=6 \%$ polyacrylamide gels without and with ethidium bromide, respectively. The white rectangle on the PA gel corresponds to the 997- and 895-bp fragments which run together, because the 895-bp fragment has a reduced electrophoretic migration $(R$ value 1.10). Lane 1, BamHI (B) + Xbal $(\mathrm{X})$; lane 2, EcoRI (E) + BamHI (B) restriction sites. 
the 3 ' end of the $d n a A$ gene, the oriC origin, and the initial sequence of the dnaN gene (Figure 1, lane 1). On PA gels, these two fragments migrate with the same mobility (Figure 1, lane 1, rectangle) because the 895-bp fragment has a reduced migration ( $R$-value 1.10 , lane 1 , white arrow). This same clone was cleaved with the enzymes EcoRI/ BamHI (Figure 1, lane 2), and the 621-bp EcoRI subfragment presented normal migration on PA gels ( $R$-value 0.90). The 290 - and the 981 -bp fragments had a significant reduction of mobility on PA gels (Figure 1, lane 2, white arrows), with $R$-values of 1.19 and 1.12 , respectively.

The 981-bp fragment (Figure 1, lane 2), which is 86-bp longer than the 895-bp fragment (Figure 1, lane 1), did not show a total return to its real molecular weight on the PA + $\mathrm{EtBr}$ gel. This effect may be due to transitory formation of the alternative cruciform structures on the left side of this segment. Structures other than bent DNA are not affected by $\mathrm{EtBr}$, which can explain the electrophoretic behavior observed for the 981-bp fragment on the PA + EtBr gel.

The p16Kori clone was cleaved with the restriction enzymes Smal + BamHI (Figure 2, lane 1). The 1300- and 803-bp fragments contain the rop promoter and $\mathrm{kan}^{r}$ gene.
The 364-bp fragment (Figure 2, lane 1, white arrow), which contains the $X$. fastidiosa oriC replication origin, presented reduced electrophoretic mobility ( $R$-value 1.12$)$.

\section{Bent structure}

The sequence of the 1892-bp fragment was analyzed with the Map15a software for helical curvature parameters, and with the 3-D15m1 software for 2-D projection. The helical parameters of ENDS ratio, $\Delta \mathrm{G}$ value, roll angle, and twist angle confirmed the presence of the bent DNA sites at the 160- (b1), 860- (b2) and 1570-bp (b3) positions (Figure 3). The highest ENDS ratio value, 1.19, was observed for the $b 1$ bent DNA site within the 290-bp fragment $(B a m H I / E c o R I)$. This site is located in the intergenic region between the $r p m H$ and $d n a A$ genes, a region with a negative roll angle $(-0.15)$ and a twist angle greater than $34.00^{\circ}$ $\left(34.08^{\circ}\right)$. The DNA bent site $b 2$ was located at the end of the 621-bp fragment. This site showed the smallest ENDS ratio value 1.13 , a twist angle of $34.11^{\circ}$, and a higher negative roll angle of -0.34 . The $X$. fastidiosa oriC replication site presented the bent DNA site $b 3$, with an ENDS ratio value of 1.16 , a roll angle of -0.77 (the most negative
Figure 2. Electrophoretic mobility analysis of the Xylella fastidiosa oriC. The restriction map of the p16Kori clone is at the top. The $R$-value of 1.12 is shown above the fragment, which is indicated on the gels by white vertical arrows; black horizontal arrows indicate the plasmid pBluescript (pBS). $M=$ molecular weight marker $1-k b$ Ladder; $\mathrm{AGA}=1.0 \%$ agarose gel; $\mathrm{PA}$ and $\mathrm{PA}+\mathrm{EtBr}=6 \%$ polyacrylamide gels without and with ethidium bromide, respectively; B = BamHI; S = Smal restriction sites.
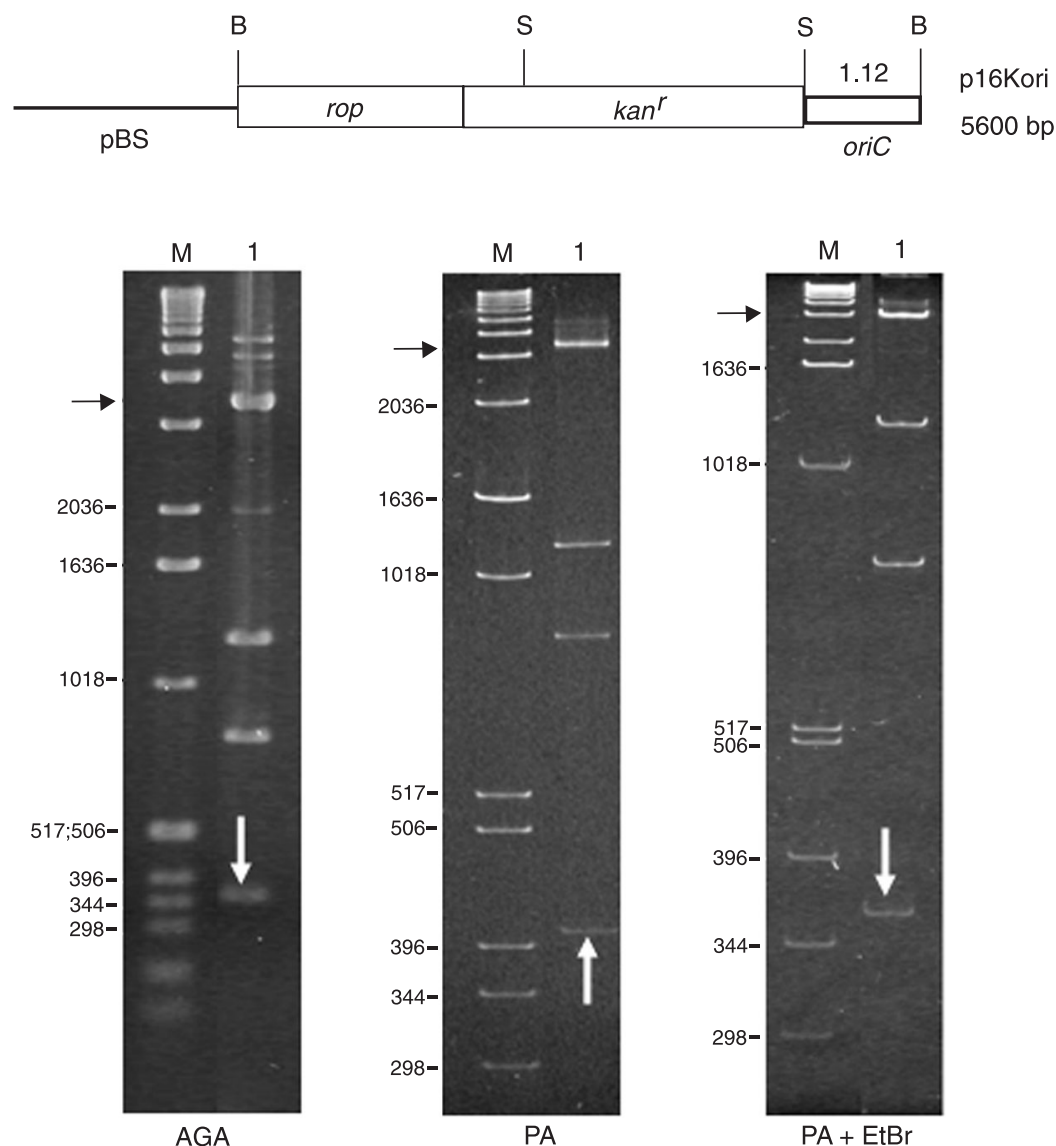

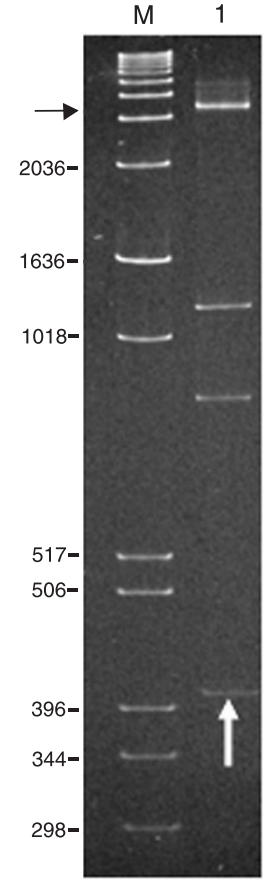

PA

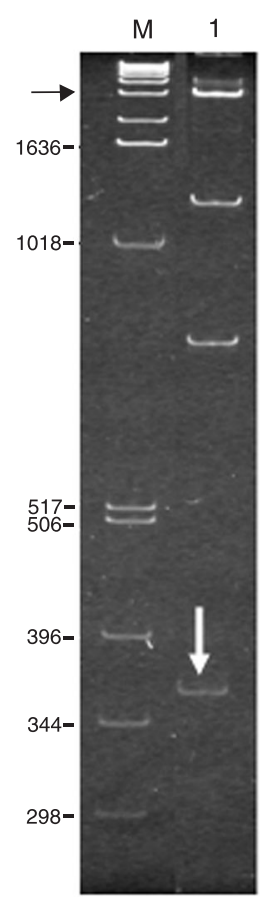

$\mathrm{PA}+\mathrm{EtBr}$ 
supercoiling value), and a twist angle greater than $34.00^{\circ}$ $\left(34.07^{\circ}\right)$. The lowest $\Delta G$ value was observed for the bent DNA site $b 3$.

Negative roll angles, that cause a narrow minor groove, suggest a bent region that, associated with a twist parameter of $>34.00^{\circ}$, indicates curved negative supercoiling DNA. This structure has been reported to have high affinity for proteins such as DnaA (31). These parameters suggest that the bent DNA site $b 3$ is actively involved in oriC activity.

The 2-D projection of the 1892-bp fragment was obtained using the 3-D15m1 software (Figure 4), which allows the visualization of the global structure of the fragment produced by the cumulative effect of the bent sites $b 1, b 2$, and $b 3$. We observed low $\Delta \mathrm{G}$ values $(227,219$ and 207, respectively), which arise from the AT-rich tracts (Figure 4, black rings). Five putative DnaA boxes (R1-R5) and the eight $A C C$ repetition box with 24 nucleotides previously reported (21) are located in this region. The rotation of the fragment sequence by $30^{\circ}$ and $60^{\circ}$ confirms that the bent sites are important for the maintenance of the shape of these fragments and should be essential for the maintenance of the function of these regions.

\section{Nucleotide sequence analysis}

Intrinsic bent DNA sites occur in regions containing tracts of adenine/thymine phased with the DNA helix turn ( 10.5 bp). The CAnT motif is located either in the center or near the curvature and seems to be responsible for high bent peak formation (7). For the analysis of the nucleotide sequences in the bent DNA sites described in the present study, we confirmed the DNA helix turn phasing of two or more adenine/thymine tracts, which were located around the sites, and the presence of the CAnT motifs. Figure 5 shows the three bent DNA sites, $b 1, b 2$, and $b 3$, in which the curvature peak is represented by a square. The $b 1$ site, located in the intergenic region between the $\mathrm{rpmH}$ and $d n a A$ genes, is flanked by CAnT motifs (double underlined). The $b 2$ site, located inside the $d n a A$ gene, is formed by phased AT tracts (underlined) and by an upstream CAnT motif (double underlined). The $b 3$ site, located at the $5^{\prime}$ end of the oriC, is also formed by $A$ and/or $T$ phased tracts (underlined). The GATC motif (Figure 5, bold and italic) was observed to be distributed in the sequence analyzed.

A detailed analysis of the oriC replication origin in $X$. fastidiosa shows the previously described AT-rich tracts with 16 nucleotides (bold), five DnaA boxes (R1-R5), and 24 nucleotides of the ACC repetition box (bold and underlined). In the present study, we identified a motif containing four tracts of six nucleotides called ATP-dependent DnaA box (Figure 5, wavy lines) (32).

\section{oriC DNA curvature}

In order to confirm the structural aspects of oriC among related prokaryotes, 2-D modeling of the 3-D DNA path, the ENDS ratio, and the roll and twist helical parameters were obtained for the nucleotide sequence of the replication origins from $X$. fastidiosa 9a5c, X. fastidiosaTemecula1, $B$. subtilis, and E. coli (33). We used the Map15a software to assess helical curvature parameters and the 3-D15m1

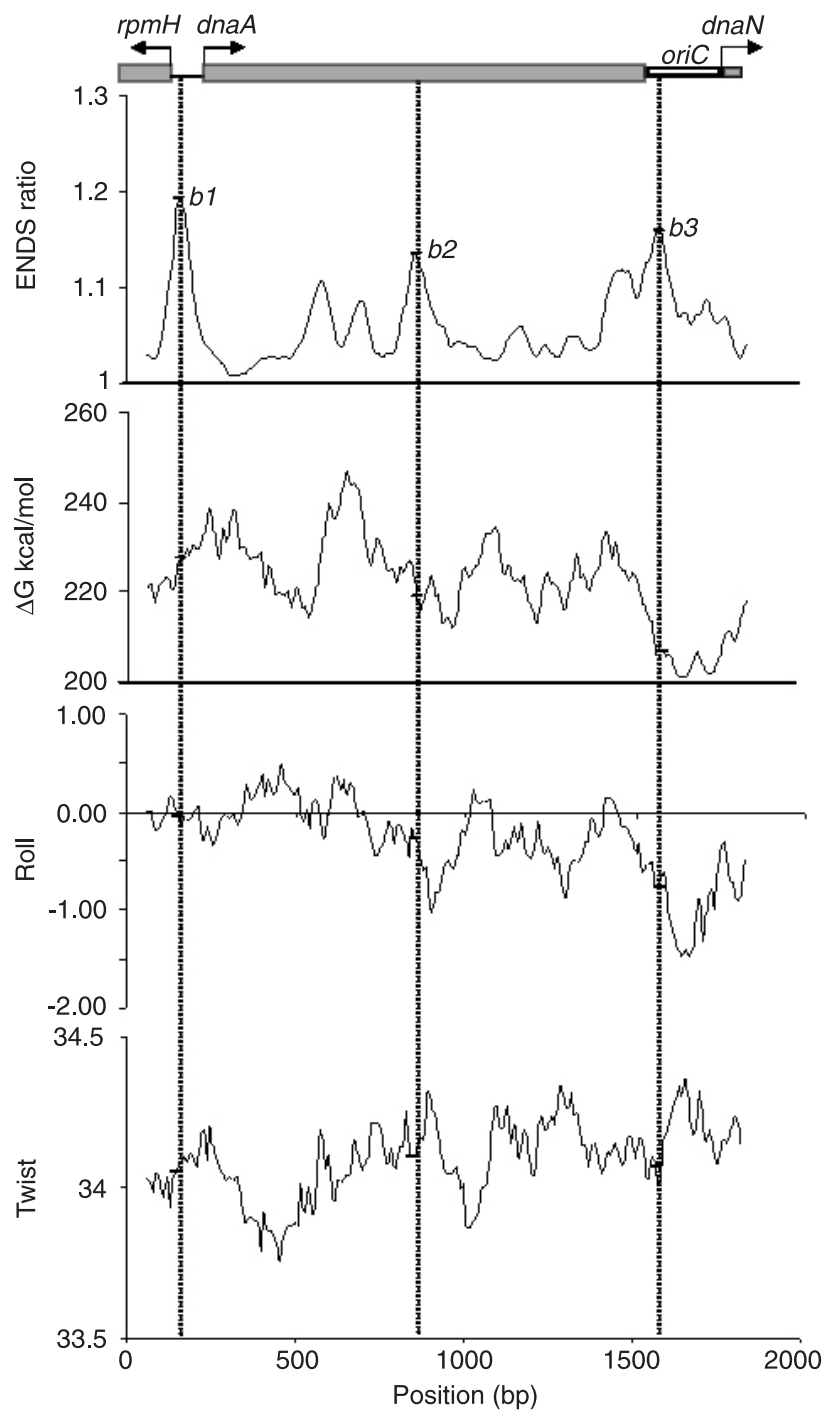

Figure 3. In silico analysis of the Xylella fastidiosa 9a5c oriC chromosomal segment. The restriction map is at the top; it contains the $r p m H$ gene initial sequence, the 141-bp intergenic region, the $d n a A$ gene, the replication origin oriC, and the $5^{\prime}$ end sequence of the $d n a N$ gene. The arrows indicate the start and direction of genetic transcription. The intrinsic bent DNA sites at the 160- (b1), 860- (b2), and 1570-bp (b3) position are shown. The Map15a software, with 120-bp window width and a 10-bp step, was used to calculate the helical parameters ENDS ratio, $\Delta G$, roll angle, and twist angle. 


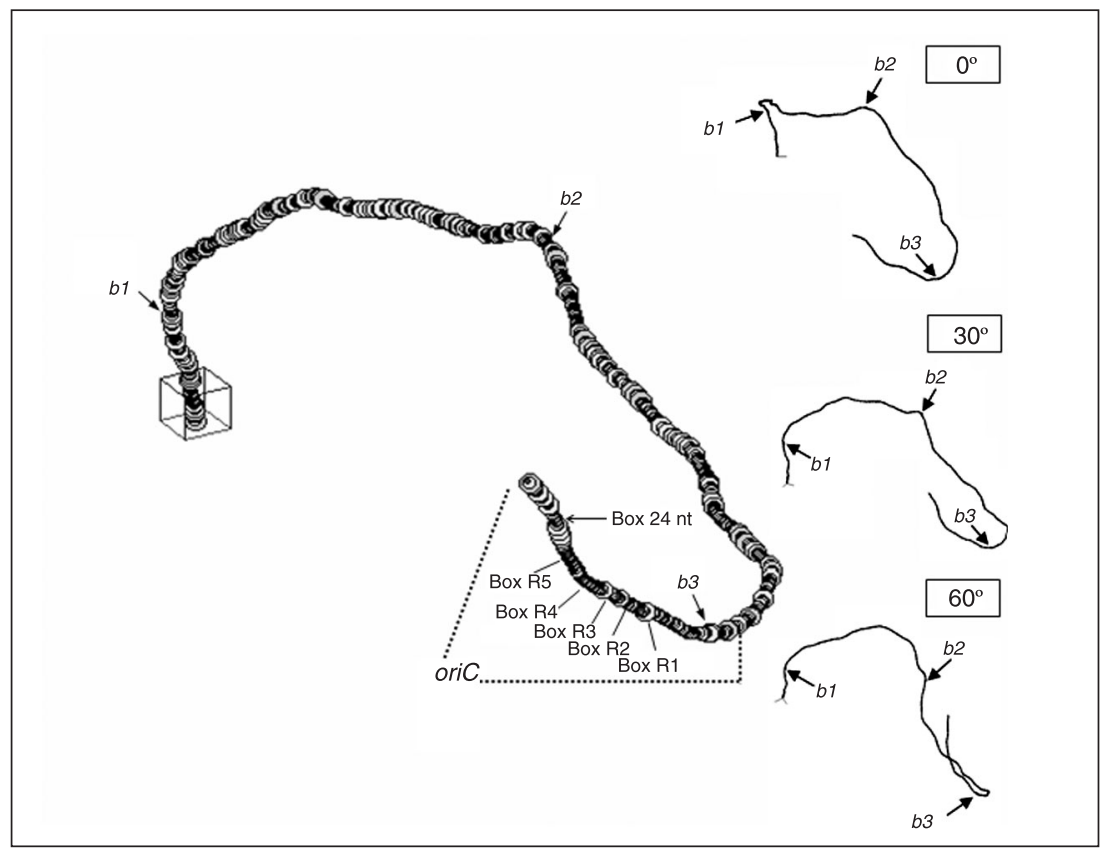

TTGATAATACGCTTGGTGGCCATCGGTTCTCTATTACACTATTTATGT AAA bl tgatagtcaattcatgatcectgagetgettegcatcatcetcgetttce aaagtgaaacagcccattactgatgacaacattg $\overrightarrow{\text { TGGA ATCTTGGTCCCGTT }}$ GCCTGGAACGTCTTGAACTGATTCCCGCCAGÅ.

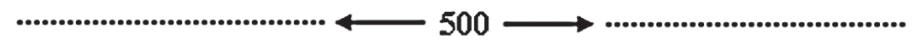
GCTACAАGATAAAGTATGGATCAGTTTAAGCGTCAGTTCCATCAА TTGACG $\overline{b 2}$

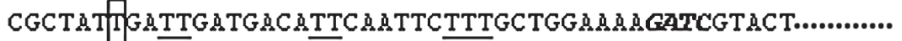

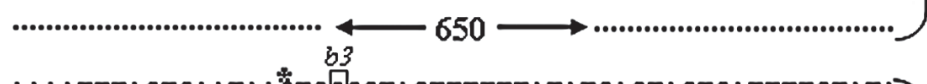

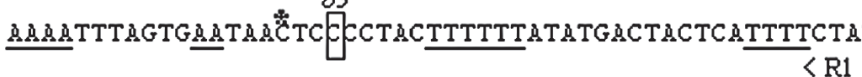
GAAATGTAAAAATAAAT TAATTCTTTCCAAGGA AGTGTAAAAACTTGTGGAT $\mathrm{R} 2>$ R3> AAA T \& GATTAAATTTTATAAAAATTTSCC\&C\&GSTTTCC\&G\&TGATC R4 >

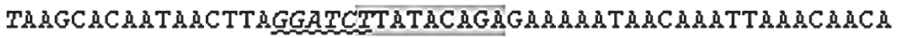
R5 >

TCTTAGAGCACTTCTCCACA A A AATTTCTACCACCACCACCACCACCACCA dnal

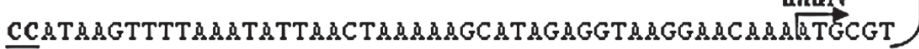

Figure 4. 2-D projection of the 3-D trajectory of the Xylella fastidiosa oriC chromosomal segment. The three intrinsic bent DNA sites, at the 160- (b1), 860- (b2), and 1570-bp (b3) positions, indicated by the of ENDS ratio (Figure 3) are shown. On the left side of the figure, rings of different sizes represent the free energy variation $(\Delta \mathrm{G})$, which predicts the stability of the double helix. The larger gray rings represent higher $\Delta \mathrm{G}$ values, and the small black rings represent lower values. The oriC replication origin is indicated by the presence of five DnaA boxes (R1-R5) and eight ACC repetition boxes of 24 nucleotides. The cube indicates the beginning of the sequence and the planned direction of the 2-D projection. The anticlockwise $30^{\circ}$ and $60^{\circ}$ rotation of these fragments, shown on the right side, confirms that the three bent DNA sites are important for the maintenance of the fragment shape. $\operatorname{dnat}$ oriC
Figure 5. Sequence analysis of the intrinsic bent DNA sites $b 1, b 2$, and $b 3$. The nucleotides on the peaks of the bent DNA sites are within the rectangle. The initial sequence of the rpmH gene (105 bp in capital letters), an intergenic region of 141 bp (lower case letters), part of the $d n a A$ gene, the replication origin segment oriC, and the initial sequence of the $d n a N$ gene (24 bp in capital letters) are indicated. Two or more adenine/thymine tracts phased with the DNA helix turn are underlined. The CAnT motif, which can be observed in the center or around the curvature, is indicated by double underlining. Two tracts of 16 AT-rich nucleotides are indicated for the oriC (in bold). Motifs of the six nucleotide consensus sequence for the ATP-dependent DnaA box are represented by wavy lines, and the location and direction of the five DnaA boxes (R1$\mathrm{R} 5$ in gray) are indicated. The ACC box with 24 nucleotides is in bold and underlined. The five GATC repetitions are in italic and bold. The oriC sequence starts 3 bp upstream of the center of the bent DNA site $b 3$ (star).

TTCAGATTACAGCGCGA 
to model the 2-D projection (Figure 6A-D, respectively). For each origin, we identified the positions of the most prominent curvature peaks with circles in the 2-D projection. The $X$. fastidiosa 9a5c origin sequence shows two main peaks in the ENDS ratio: 1.06, located before the first DnaA box at the 36-bp position, and 1.07, located between the first and second DnaA boxes at the 117-bp position (Figure 6A). The observed ENDS ratios for the $X$. fastidiosa Temecula1 origin were: 1.06 , located before the first DnaA box at the 36-bp position, and 1.07 located within the second DnaA box at the 117-bp position (Figure 6B). For $B$. subtilis, we identified two prominent ENDS ratio peaks located at positions 90 and $147 \mathrm{bp}$. These two peaks show the same ENDS ratio value of 1.04 , with the first located between the second and third DnaA boxes and the second located after the third DnaA box (Figure 6C). The E. coli origin (Figure 6D) shows three prominent ENDS ratio peaks with 1.03 values, as well as two others with a value of 1.02 . The first, at the $90-\mathrm{bp}$ position, is located between the second and third DnaA boxes. The other four peaks (at the 183-, 234-, 270-, and 315-bp positions) are located after the third DnaA box.

The replication origins of these four organisms were analyzed by the roll and twist helical parameter values (Figure 6A-D). There is a predominance of negative roll angle, in addition to twist values above $34.00^{\circ}$, at the same position as the ENDS ratio peaks described for all oriC analyzed.

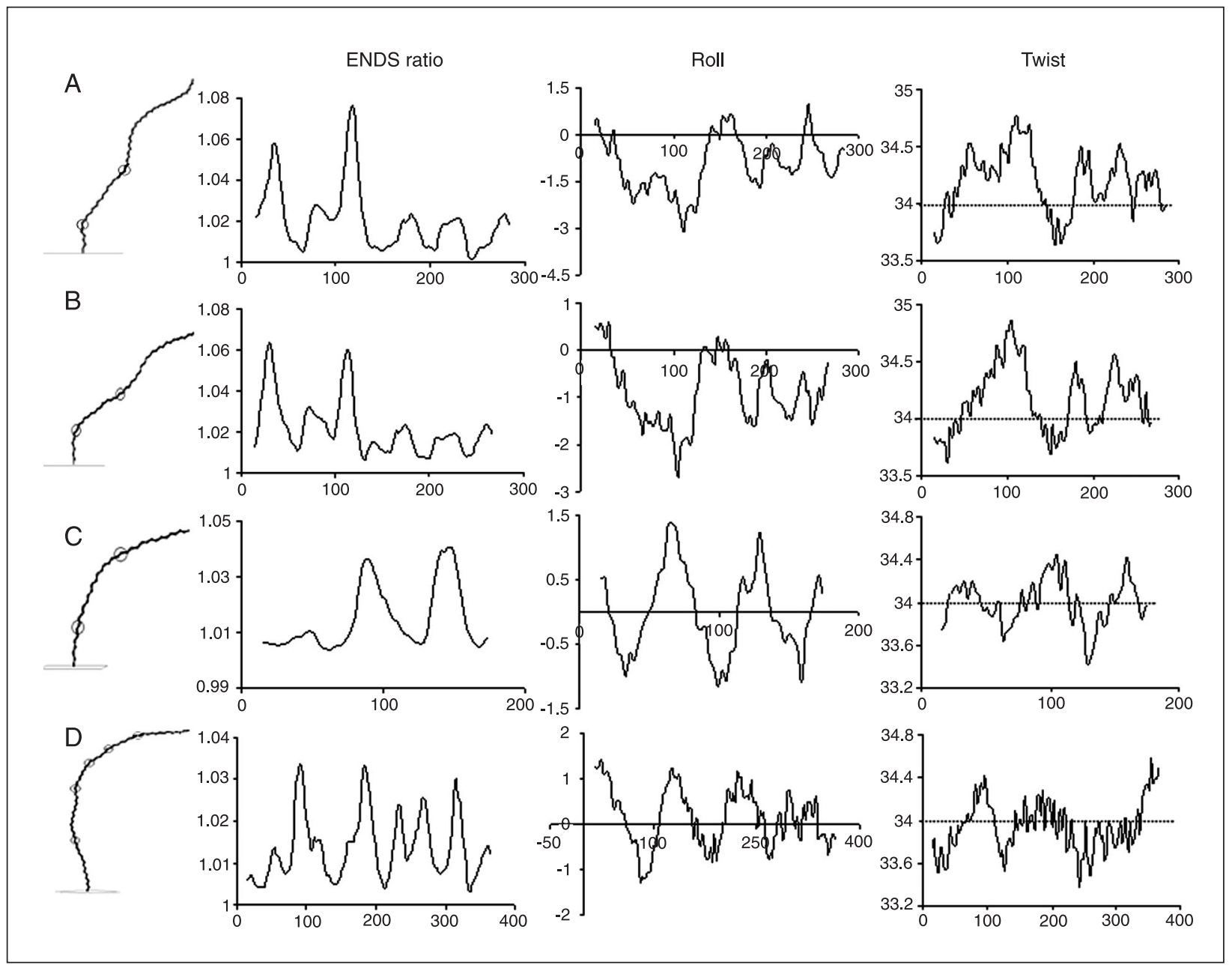

Figure 6. In silico analysis of the bacterial oriC. The nucleotide sequences from Xylella fastidiosa 9a5c (A), X. fastidiosa Temecula1 (B), Bacillus subtilis (C), and Escherichia coli (D) are described in DoriC, a database of the oriC region in bacterial genomes (33). The 3-D15m1 software was used for 2-D projection, and the helical curvature parameters (ENDS ratio, twist angle, and roll angle) were calculated using the Map15a software with a 60-bp window width and a 10-bp step. The ENDS ratio positions are indicated by a circle in the 2-D projection. 


\section{Discussion}

Electrophoretic and in silico analysis revealed that the 1892-bp fragment analyzed in the present study, which contains the chromosomal replication origin (oriC) of $X$. fastidiosa, showed intrinsic bent DNA sites. The $R$-values $(1.19,1.12$, and 1.10) are in accordance with all described bent DNA sites, which are greater than or equal to 1.10 (34). The electrophoretic behavior of these fragments is consistent with the 3-D modeling, which shows the presence of sequence-directed curvature.

In silico analysis of this fragment showed at least three bent sites at the 160- (b1), 860- (b2), and 1570-bp (b3) positions, all with ENDS ratio values higher than 1.11. The $b 1$ site is located in the promoter region of the $d n a A$ and $r p m H$ genes, and can play a role in the transcription of these genes. The $b 2$ site is located inside the $d n a A$ gene. There is no explication for this location, since bent DNA sites have only been described in promoters, intronic regions, and replication origins (14). The $b 3$ site is located in the replication origin of $X$. fastidiosa and may be involved in the replication process of this bacterium's chromosomal DNA. Regarding the bent DNA site $b 3$, its relationship with the dnaN gene promoter is difficult to establish because it is located -293 bp from the starting point of this gene. It has been reported that a bent DNA site upstream of the prokaryotic promoter is always located at -50 to $-200 \mathrm{bp}(30)$.

ENDS ratio values of 1.15 or higher were defined as indicative of bent DNA elements in the Bacillus thuringiensis chromosome, for which an average of eight ENDS ratio values yielding a mean greater than 1.2 for each $10-\mathrm{kb}$ segment has been reported (35). However, ENDS ratio values lower than 1.10 were reported for the Xenopus laevis ribosomal intergenic sequence (36). On the basis of these results, it is clear that the ENDS ratio value contributes importantly to fragment curvature. Several peaks with lower ENDS ratios can determine a strongly curved segment. The length of the observed fragment and the calculation parameters, such as the window width and the interval steps, contribute to the establishment of the ENDS ratio value (30).

The helical parameters twist and roll angles were also evaluated for the replication origin of three additional bacterial oriC segments. We found negative roll angles ranging from -0.15 to -0.76 for this region, as well as twist angles ranging from $34.07^{\circ}$ to $34.11^{\circ}$. These data show negative roll angles and twist angles above $34.00^{\circ}$, in agreement with curved regions with negative supercoiling, as described for regions with high affinity for the DnaA protein (31). According to Molina et al. (37), bending and supercoiling are requirements for the creation of the unique structure of oriC that allows the cell to initiate DNA replication correctly and at the proper time. Furthermore, it has been reported that the SeqA protein in bacterial cells, which represses DNA replication activity, promotes helical changes in DNA structure that cause positive supercoiling of the segment (38).

To support studies about bent DNA structure, atomic force microscopy has recently been performed in the M13 plasmid replication origin (39), providing independent evidence that this replication origin contains a stable bend.

The analysis of the 1892-bp fragment of the $X$. fastidiosa nucleotide sequence shows two or more adenine/ thymine tracts phased with the helical turn, located around the bent DNA sites. Similarly, the CAnT motif can also be found in DNA sequence-directed curvatures (40). Sequence analyses of the three bent sites revealed a predominance of stretches of multiple poly (dA) and poly (dT) tracts around the bent sites, showing a periodicity of $\sim 10$ bp or multiples of this value. It is also possible to observe five GATC motifs, two of which are located in the replication origin (oriC). It has been reported that the GATC motif also contributes to intrinsic DNA curvature at oriC, and the degree of bending is modulated by methylation (37). Furthermore, we also identified tracts of six nucleotides, named ATP-dependent DnaA box (32).

The results reported here show that the oriC of the related prokaryotes $X$. fastidiosa $9 \mathrm{a} 5 \mathrm{c}$ and $X$. fastidiosa Temecula1 show great similarities in both their 2-D trajectories and helical parameters for curvature. In the other two bacterial oriC analyzed here, from $B$. subtilis and $E$. coli, both the value of the ENDS ratio peaks and the 2-D trajectory of the curvature increased.

The present study demonstrated that the $X$. fastidiosa $9 \mathrm{a} 5 \mathrm{c}$ oriC has particular features such as intrinsic bent DNA sites which induce a curved DNA structure in this region. This structure is probably involved in the initiation of DNA replication for this organism. Furthermore, in silico analysis of three related bacterial oriC sequences showed similar negative roll angles, as well as twist values greater than $34.00^{\circ}$. A particular 2-D trajectory was observed for each replication initiation segment.

\section{Acknowledgments}

We thank Valmir Peron and Marli Licero Schuete Silva for their dedicated technical assistance. 


\section{References}

1. Watson JD, Crick FH. Molecular structure of nucleic acids; a structure for deoxyribose nucleic acid. Nature 1953; 171: 737-738.

2. Marini JC, Levene SD, Crothers DM, Englund PT. A bent helix in kinetoplast DNA. Cold Spring Harb Symp Quant Biol 1983; 47 (Part 1): 279-283.

3. Zhang Y, Xi Z, Hegde RS, Shakked Z, Crothers DM. Predicting indirect readout effects in protein-DNA interactions. Proc Natl Acad Sci U S A 2004; 101: 8337-8341.

4. Goodsell DS, Dickerson RE. Bending and curvature calculations in B-DNA. Nucleic Acids Res 1994; 22: 5497-5503.

5. Fujimura FK. Point mutation in the polyomavirus enhancer alters local DNA conformation. Nucleic Acids Res 1988; 16: 1987-1997.

6. Jauregui R, Abreu-Goodger C, Moreno-Hagelsieb G, Collado-Vides J, Merino E. Conservation of DNA curvature signals in regulatory regions of prokaryotic genes. Nucleic Acids Res 2003; 31: 6770-6777.

7. Fiorini A, Basso LR Jr, Paco-Larson ML, Fernandez MA. Mapping of intrinsic bent DNA sites in the upstream region of DNA puff BhC4-1 amplified gene. J Cell Biochem 2001; 83: 1-13.

8. Virstedt J, Berge T, Henderson RM, Waring MJ, Travers AA. The influence of DNA stiffness upon nucleosome formation. J Struct Biol 2004; 148: 66-85.

9. Iarovaia OV, Borounova V, Vassetzky YS, Razin SV. An unusual extended DNA loop attachment region is located in the human dystrophin gene. J Cell Physiol 2006; 209: 515521.

10. Palin AH, Critcher R, Fitzgerald DJ, Anderson JN, Farr CJ. Direct cloning and analysis of DNA sequences from a region of the Chinese hamster genome associated with aphidicolinsensitive fragility. J Cell Sci 1998; 111 (Part 12): 1623-1634.

11. Pasero P, Sjakste N, Blettry C, Got C, Marilley M. Longrange organization and sequence-directed curvature of $\mathrm{Xe}$ nopus laevis satellite 1 DNA. Nucleic Acids Res 1993; 21: 4703-4710.

12. Altman AL, Fanning E. Defined sequence modules and an architectural element cooperate to promote initiation at an ectopic mammalian chromosomal replication origin. Mol Cell Biol 2004; 24: 4138-4150.

13. Fiorini A, de Gouveia FS, de Soares MA, Stocker AJ, Ciferri $\mathrm{RR}$, Fernandez MA. DNA bending in the replication zone of the C3 DNA puff amplicon of Rhynchosciara americana (Diptera: Sciaridae). Mol Biol Rep 2006; 33: 71-82.

14. Fiorini A, Gouveia FS, Fernandez MA. Scaffold/Matrix Attachment regions and intrinsic DNA curvature. Biochemistry 2006; 71: 481-488.

15. Brylawski BP, Cohen SM, Cordeiro-Stone M, Schell MJ, Kaufman DG. On the relationship of matrix association and DNA replication. Crit Rev Eukaryot Gene Expr 2000; 10: 91 99.

16. Myllykallio H, Lopez P, Lopez-Garcia P, Heilig R, Saurin W, Zivanovic $Y$, et al. Bacterial mode of replication with eukaryotic-like machinery in a hyperthermophilic archaeon. Science 2000; 288: 2212-2215

17. Cvetic C, Walter JC. Eukaryotic origins of DNA replication: could you please be more specific? Semin Cell Dev Biol 2005; 16: 343-353.
18. Eckdahl TT, Anderson JN. Computer modelling of DNA structures involved in chromosome maintenance. Nucleic Acids Res 1987; 15: 8531-8545.

19. Chang CJ, Garnier M, Zreik L, Rossetti V, Bové JM. Culture and serological detection of the xylem-limited bacterium causing citrus variegated chlorosis and its identification as a strain of Xylella fastidiosa. Curr Microbiol 1993; 27: 137142.

20. Simpson AJ, Reinach FC, Arruda P, Abreu FA, Acencio M, Alvarenga $R$, et al. The genome sequence of the plant pathogen Xylella fastidiosa. The Xylella fastidiosa Consortium of the Organization for Nucleotide Sequencing and Analysis. Nature 2000; 406: 151-159.

21. Monteiro PB, Teixeira DC, Palma RR, Garnier M, Bove JM, Renaudin J. Stable transformation of the Xylella fastidiosa citrus variegated chlorosis strain with oriC plasmids. Appl Environ Microbiol 2001; 67: 2263-2269.

22. Ogasawara N, Yoshikawa H. Genes and their organization in the replication origin region of the bacterial chromosome. Mol Microbiol 1992; 6: 629-634.

23. Sambrook J, Russell DW. Molecular cloning: A laboratory manual. 3rd edn. New York: Cold spring Harbor Laboratory Press; 2001

24. Del Sal G, Manfioletti G, Schneider C. The CTAB-DNA precipitation method: a common mini-scale preparation of template DNA from phagemids, phages or plasmids suitable for sequencing. Biotechniques 1989; 7: 514-520.

25. Diekmann S, Wang JC. On the sequence determinants and flexibility of the kinetoplast DNA fragment with abnormal gel electrophoretic mobilities. J Mol Biol 1985; 186: 1-11.

26. Milot E, Belmaaza A, Wallenburg JC, Gusew N, Bradley WE, Chartrand $\mathrm{P}$. Chromosomal illegitimate recombination in mammalian cells is associated with intrinsically bent DNA elements. EMBO J 1992; 11: 5063-5070.

27. Bolshoy $A$, McNamara $P$, Harrington RE, Trifonov EN. Curved DNA without A-A: experimental estimation of all 16 DNA wedge angles. Proc Natl Acad Sci U S A 1991; 88: 2312-2316.

28. Delabre ML, Pasero P, Marilley M, Bougis PE. Promoter structure and intron-exon organization of a scorpion alphatoxin gene. Biochemistry 1995; 34: 6729-6736.

29. Marilley M, Pasero P. Common DNA structural features exhibited by eukaryotic ribosomal gene promoters. Nucleic Acids Res 1996; 24: 2204-2211.

30. Calladine CR, Drew HR, Luisi BF, Travers AA. Understanding DNA: The molecule and how it works. 3rd edn. Italy: Elsevier Academic Press; 2004.

31. Erzberger JP, Mott ML, Berger JM. Structural basis for ATP-dependent DnaA assembly and replication-origin remodeling. Nat Struct Mol Biol 2006; 13: 676-683.

32. Robinson NP, Bell SD. Origins of DNA replication in the three domains of life. FEBS J 2005; 272: 3757-3766.

33. DoriC: a database of oriC region in bacterial genomes. http:/ /tubic.tju.edu.cn/doriC/. Accessed August 21, 2007.

34. Anderson JN. Detection, sequence patterns and function of unusual DNA structures. Nucleic Acids Res 1986; 14: 85138533.

35. VanWye JD, Bronson EC, Anderson JN. Species-specific patterns of DNA bending and sequence. Nucleic Acids Res 
1991; 19: 5253-5261.

36. Roux-Rouquie M, Marilley M. Modeling of DNA local parameters predicts encrypted architectural motifs in Xenopus laevis ribosomal gene promoter. Nucleic Acids Res 2000; 28: 3433-3441.

37. Molina F, Skarstad K. Replication fork and SeqA focus distributions in Escherichia coli suggest a replication hyperstructure dependent on nucleotide metabolism. Mol Microbiol 2004; 52: 1597-1612.
38. Klungsoyr HK, Skarstad K. Positive supercoiling is generated in the presence of Escherichia coli SeqA protein. Mol Microbiol 2004; 54: 123-131.

39. Lu Y, Weers BD, Stellwagen NC. Analysis of the intrinsic bend in the M13 origin of replication by atomic force microscopy. Biophys J 2003; 85: 409-415.

40. Crothers DM, Haran TE, Nadeau JG. Intrinsically bent DNA. J Biol Chem 1990; 265: 7093-7096. 